\title{
SECURITY ISSUES AND VARIOUS ATTACKS IN WIRELESS SENSOR NETWORK: A SURVEY
}

\author{
S. NagaMallik Raj ${ }^{1}$, Divya Midhunchakkaravarthy ${ }^{1}$ and Debnath Bhattacharyya ${ }^{2}$ \\ ${ }^{1}$ Department of Computer Science and Multimedia, \\ Lincoln University College, Malaysia \\ ${ }^{2}$ Department of Computer Science and Engineering, \\ Vignan's Institute of Information Technology, \\ Visakhapatnam-530049, India \\ \{nagamallik.Sappa,divya\}@lincoln.edu.my, debnathb@gmail.com
}

\begin{abstract}
Wireless sensor networks is a rising field towards innovation, because of its numerous utilizations that has lead to the improvement of modest, expendable and independent battery fueled PCs, known as sensor hubs or "bits". The testing of some portion of a remote sensor system makes the security have extreme limitations than customary systems. In this paper we explore security issues that are present in the WSN alongside a few Attacks in WSN[4]. We recognize these security dangers and would like to propose this survey on security systems for remote sensor systems. Likewise, we will also look into the different security issues present in every layer.
\end{abstract}

Keywords - Network Attacks, Security, Sensor and WSN.

\section{INTRODUCTION}

In correspondence to innovation of networks, at least two gadgets should be associated through an interface to transfer the data[1].

\subsection{COMPUTER NETWORK}

An arrangement of PC's, additionally alluded to as a set of computers with organized information, is a progression of interconnected hubs that can transmit, get and trade information.

\subsection{TYPES OF NETWORK}

a) Wired Networks: If all the communication devices are connected with the help of wires, then it is called as Wired networks.

b) Wireless Networks: If the communication devices were connected without wires, then it is called as Wireless networks.

\section{WHY USE WIRELESS NETWORKS?}

In remote systems, we can effectively traverse from one spot then onto the next spot such that it does not require any wires. It can deal with an enormous number of gadgets to build up a system. We can include additional gadgets all around the existing system effectively. Overall this consumes less time with low costs[9][11][12].

Received: May 10, 2019

Reviewed: August 12, 2019

Accepted: September 2, 2019 


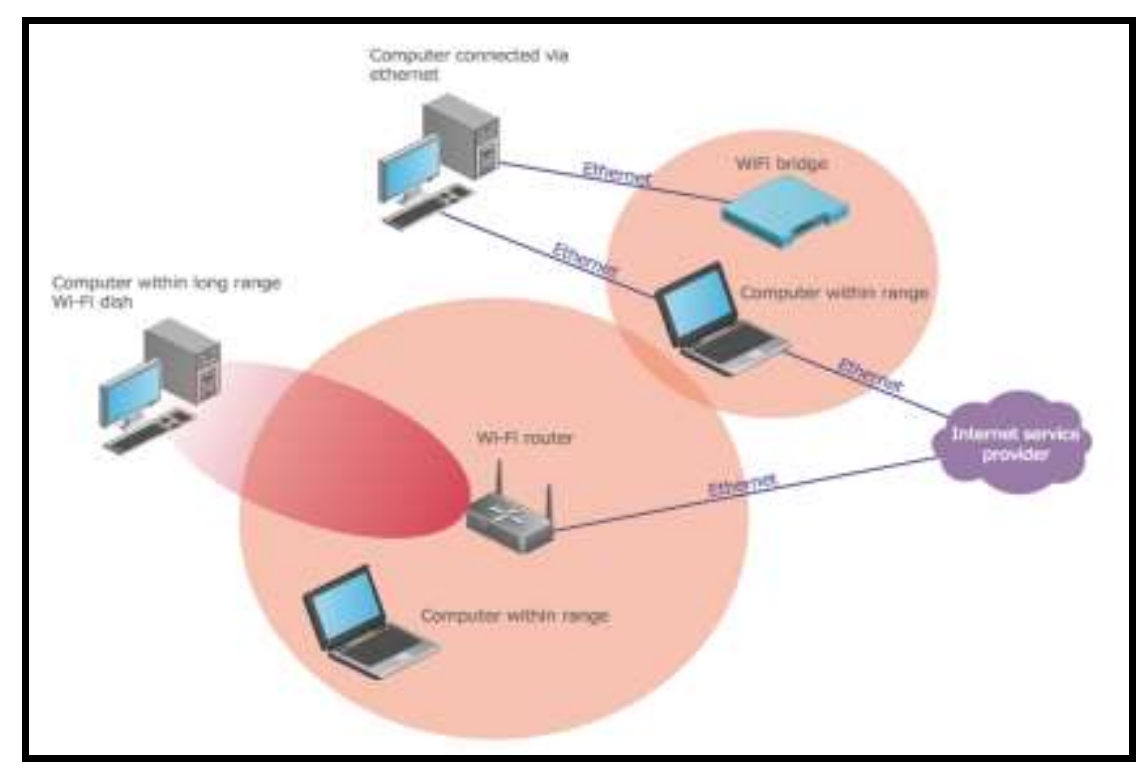

Fig. 1 Communications in Wireless Networks [1]

\subsection{WIRELESS SENSOR NETWORKS}

A remote sensor and actuator system is a collection of little arbitrarily scattered gadgets that give few fundamental capacities like; the capacity to work with gadgets, for example- switches, engines or actuators that control those conditions; the capacity to give productive, solid interchanges through a remote system and more[9].

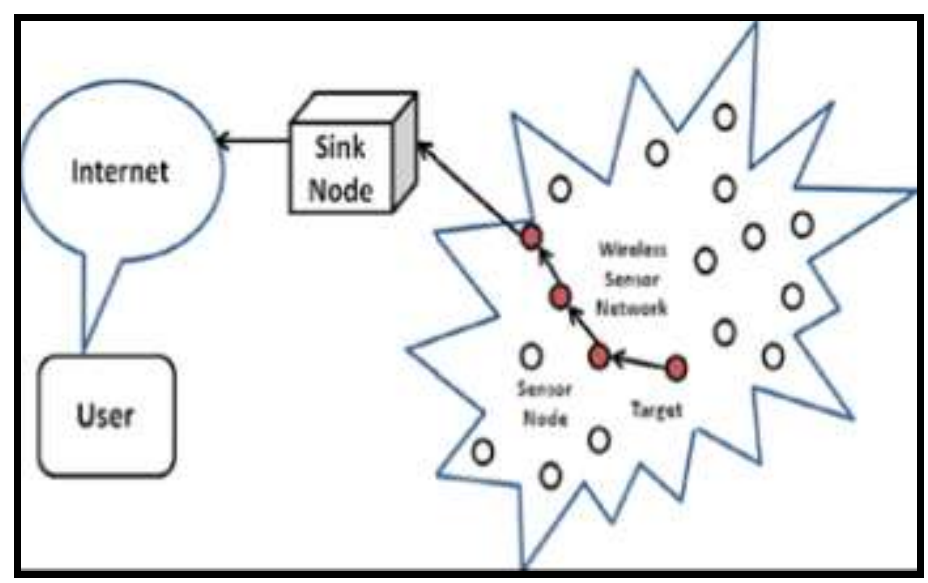

Fig. 2 Wireless sensor network [9]

Remote sensor systems utilize three essential systems administration topologies; pointto-point, star or mesh. Point-to-point is essentially a devoted connection between two points. Star systems are a total of point-to-point joins, with a focal ace hub [13][14]. In the work topology, each hub has numerous pathways to every other hub, giving the most versatility and adaptability[5]. 


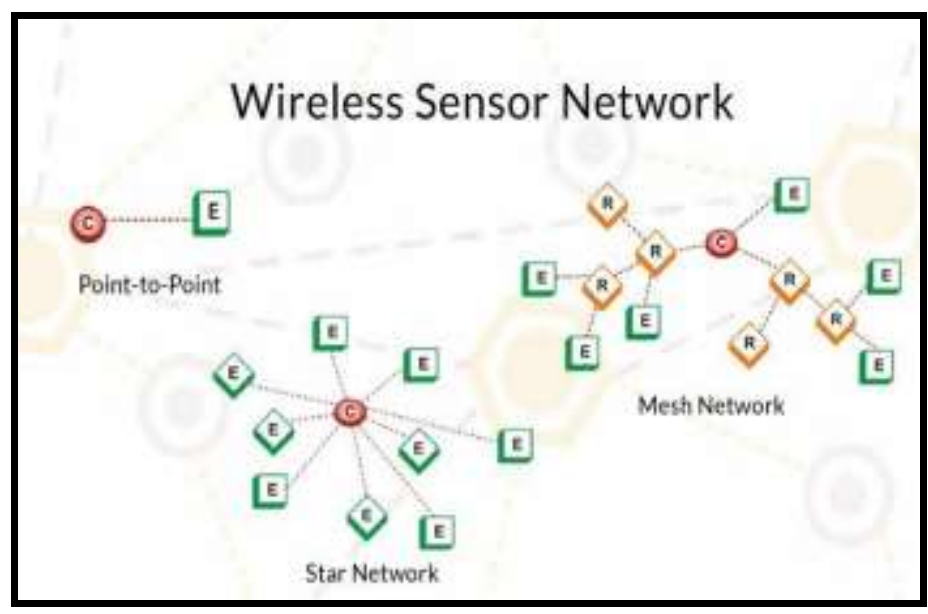

Fig. 3 Wireless sensor networks[2]

\section{IMPERATIVES INFLUENCING SECURITY IN WSNS}

Imperatives that make customary security illogical in WSNs are:

- Low vitality control: The vitality of the hubs is constrained (restricted battery lifetime), by large indispensable and no recharging batteries. Conventions of WSNs must focus principally on the protection of vitality.

- Constrained memory and calculation limit: In most of WSNs, hubs are not ready to remember keys of noteworthy size, or to complete complex conventions cryptographically [1][7][9]. Subsequently, new safety efforts are expected to address imperatives of WSNs.

\section{VITALITY FOR SECURITY}

Lifetime hub is commonly restricted by the lifetime of a minor battery, so vitality is the essential asset limitation [7][9].

- Calculation vital for the elements of security; for example- figuring, decoding, confirmation of the mark.

- Energy vital for the transmission and the board of security materials (keys, and so on).

- Energy vital for the capacity of the keys.

The test is to limit the utilization of the vitality along with amplifying the exhibitions of security. Vitality is a significant factor to think about when planning safety efforts for WSNs, preserving hub vitality to expand the lifetime, and drawing out system functionalities [15].

\section{SECURITY GOALS}

\subsection{DATA CONFIDENTIALITY}

The necessity of secrecy is required to guarantee that personal or important data is absolutely protected and not uncovered to unapproved outsiders. In view of the affectability of the information stolen, a foe may cause serious harm since he can utilize the detected information for some illicit purposes, for example- harming, blackmail, extortion [2-4]. On the off chance that we consider a third party listening stealthily to one's personal information, to be a system level risk, at that point a nearby dimension danger could be traded off hub that a foe now currently possesses. Trading off hubs are a 
major danger to privacy since the enemy could take basic information and put away on hubs, for example- cryptographic keys that are utilized to encode the correspondence.

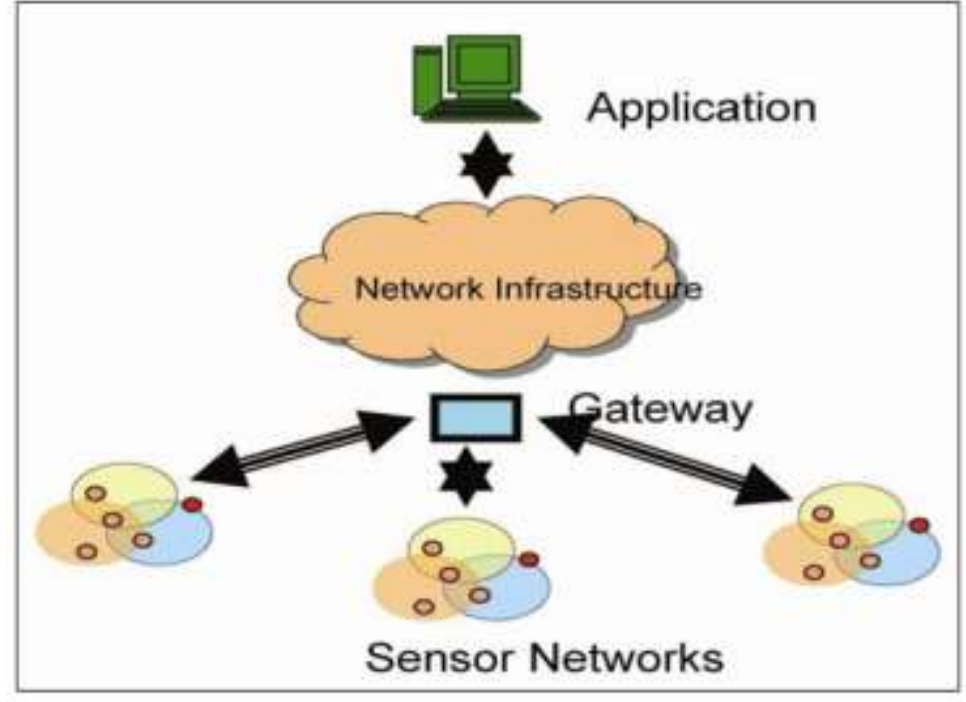

Fig. 4 Data Confidentiality [3]

\subsection{VALIDNESS AND INTEGRITY}

There is a chance that when we trade our data through unreliable systems, the data may be changed. In the event that we, without realizing this issue, utilize this modified information, then the application will be highly risky [6][8].

\subsection{ACCESSIBILITY}

Absence of accessibility may influence the task of numerous basic ongoing applications like those in the social insurance part that require an everyday activity that could even outcome in the death toll. In this way, it is basic to guarantee versatility to assaults focusing on the accessibility of the framework and discover approaches to fill in the hole made by the catching or disablement of a particular hub by allotting its obligations to some different hubs in the system [3][4].

\subsection{SECURE MANAGEMENT}

In the sensor systems, to keep up steering the data, we need secure administration.

\subsection{DATA FRESHNESS}

We have to ensure that the freshness of each message. i.e to take care that no old messages have been repeated. To ensure data freshness, inside the packet we have to add time related counter.

\subsection{ROBUSTNESS AND SURVIVABILITY}

This sensor system must be healthy along with unique security strikes. However if trap occurs as obvious or at least one of the hubs are a good yet suspicious deal, the security of the whole system ought not be broken.

\subsection{NON-RENOUNCEMENT}

The non-disavowal security estimation offers means to foreseeing a solitary or component from denying having played out a particular development related to data by making open check of various framework related exercises, (for instance, proof of 
responsibility, reason, or obligation; affirmation of data beginning stage, affirmation of ownership, confirmation of advantageous usage). It ensures the openness of affirmation that may be acquainted with an outcast and used to show that an event or movement has happened.

\subsection{TIME SYNCHRONIZATIONS}

The goal of time synchronization is to equalize the local instances for all nodes within the network, if required, considering the fact that WSNs are restrained in computation functionality, bandwidth, electricity sources and storage potential.

\subsection{SECURE LOCALIZATION}

In most sensors, there is a need to arrange the position data correctly every now and then without human mediation so that they can savage it effectively. However, an assailant can control non ensured position data without trouble through detailing phony sign qualities and replay the signal.

\subsection{ACCESS CONTROL}

Getting an access to control security degree is a guarantee against the risk of unapproved usage of framework resources. Access control ensures that simply endorsed work power or devices are allowed access to framework segments, spared information, information streams, organizations and demands. Moreover, Role-Based Access Control gives varying access levels for confirmation that individuals and units can simply gain admittance to and perform activities on, framework segments, spared information, and information streams that they are affirmed for.

\section{ATTACKS IN WSN NETWORK}

An assortment of assaults against WSNs is reported in the writing. To confront these assaults, different estimations were proposed. We present in the continuation, the foremost kinds of assaults, and in segment 9 we allot these assaults to the layers worried of the OSI model. An order of the assaults comprises in recognizing the inactive assaults from the dynamic assaults[3][6][8] .

Altering: In this an Attacker will get to the hub, to recuperate the keys which are utilized for encryption.

Dark gap: A vindictive hub occupies the directing data, with the end goal that no information will go to sink hub, to made dark gap.

Specific sending: In this assault the malevolent hub will not exchange significant information, but instead drops the essential messages.

Sybil assault: So as to participate in circulated calculations, like the decision, from the others hubs, assailant can utilize the characters.

HELLO flood assault: In systems discovering the neighbor hub, we exchange "HELLO" bundle; yet an aggressor will flood the system by sending a similar message, with the end goal of that to anticipate the trade of recognizing the steering data.

Sticking: On the recurrence groups by sending pointless data, sticking may happen, which might be brief or changeless.

Wormhole assault: The assailants here are intentionally placed at various closures of a system. They can get messages and replay them in numerous elements by way of methods for a passage [9]. 


\subsection{ASSAILANT MODEL}

The objective of an assailant (foe) is to illicitly acquire keys to put away in hubs by vulnerabilities abuse.

Solid aggressor: The enemy is considered to be present during the arrangement of hubs. They can oversee every one of the interchanges, anyplace, and at any minute.

\section{ISSUES OF SECURITY IN EACH LAYER}

In this segment, there exists layers that is put together by grouping of characterized assaults with respect to the OSI model portrayed previously.

\section{Physical layer}

This layer deals with particular frequency groups. This layer must guarantee the procedures of emanation, gathering and balance of information in a strong manner. The assaults related to the physical layer are not that many, but in the meantime; sticking onto a similar recurrence that the system utilizes, and the physical assault of a hub, can be the most hard to avoid.

Table I. Security Attacks on Each Layer of the Internet Model [3][5][6][8]

\begin{tabular}{|l|l|}
\hline \multicolumn{1}{|c|}{ Layer } & \multicolumn{1}{c|}{ Attacks } \\
\hline Application layer & Data corruption \\
\hline Transport layer & Session hijacking, SYN flooding \\
\hline Network layer & $\begin{array}{l}\text { Wormhole, black hole, Byzantine, flooding, resource } \\
\text { consumption, location disclosure attacks }\end{array}$ \\
\hline Data link layer & $\begin{array}{l}\text { Traffic analysis, monitoring, disruption MAC (802.11), } \\
\text { WEP weakness }\end{array}$ \\
\hline Physical layer & Jamming, interceptions, eavesdropping \\
\hline Multi-layer attacks & DOS, impersonation, replay, man-in-the-middle \\
\hline
\end{tabular}

\section{Link layer assaults}

This layer deals with the access to the radio channel (MAC layer), and control blunders. The enemy can just actuate crash in one byte of a transmission to ruin the whole information parcel. This then commits the unfortunate casualty hub to retransmit the information parcel and cause a passing of this hub by devouring its vitality (weariness assault). The avoidance of these assaults can be restricted to force the utilization of little parcels, and to use systems of revision to request the retransmission of bundle.

\section{Network layer assaults}

WSNs direct the bundles towards the goal, and some of the assaults in this layer are as follows: dark opening, particular sending, Sybil assault, HELLO flood assault, wormhole, and Identity replication assault. In a progressive system of sensors, the guardians hubs can check the character of the wellspring of a parcel in travel.

\section{Transport layer assaults}

The dreams of TCP, like Transport layer conventions in WSN, comprise the putting in place of begin to finish affiliation, give up to-give up strong conveyance of bundles, 
stream manipulate, clog manage, and clearing of start to complete affiliation. Like TCP conventions inside the Internet, the versatile hub is defenseless in opposition to the remarkable SYN flooding attack or session commandeering attacks [1] [3] [4].TCP multiplicatively diminishes its clog window after encountering misfortunes, which corrupts organized execution.

\section{CONCLUSION AND FUTURE WORK}

Currently, the popularity of WSN increases with the consideration of numerous analysts. This paper reviews the security challenges present in WSNs, which vary from the specially appointed systems with increasingly extreme limitations as far as vitality, calculation capacities and interchanges. Subsequently, the arrangements of security should thus be adjusted in this way. On the off chance that we know how the assailant will assault the systems, we can take preventive measures in every aspect and build up better systems for what's to come. In future work, we will work out every single imaginable answer for a wide range of assaults, with the end goal that we can build up better protected systems.

\section{REFERENCES}

[1] Gaurav Sharma, S. Bala, A.K. Verma, "Security Frameworks for Wireless Sensor Networks-I Review", Procedia Technol., vol. 6, pp. 978-987, 2012.

[2] N.S. Fayed, E.M. Daydamoni, A. Atwan, "Efficientcombined security system for wireless sensor network", Egyptian Informatics Journal., 2012.

[3] O. Amara Said, R. Beghdad, Mourad Oussalah, "Securing Wireless Sensor networks: A survey", Taylor \& Francis, feb 2013.

[4] Some issues and challenges of Wireless Sensor Networks Himani Chawla CSE Dept. Maharishi markandeshwar university Ambala, Haryana, India Volume 4, Issue 7, July 2014 ISSN: 2277 128X International Journal of Advanced Research in Computer Science and Software Engineering

[5] Sert SA, Onur E, Yazici A (2015) Security $\breve{A} \Sigma \breve{A c U ̆ E}$ and countermeasures in Surveillance Wireless

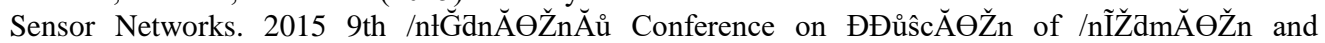
CŽmmZnŝcĂӨŽn Technologies (AICT), Russia.

[6] Security Issues in Wireless Sensor Networks: Attacks and Countermeasures Kahina CHELLI Proceedings of the World Congress on Engineering 2015 Vol I WCE 2015, July 1 - 3, 2015, London, U.K.

[7] Yadav S, Yadav RS (2016) A review on energy ĞkcŝĞnt protocols in wireless sensor networks. Wirel Netw 22: 335-350.

[8] Security requirements in Wireless Sensor Networks By Ravi Bandakkanavar | September 26, 2016

[9] Anju Bala ,Security Attacks and Challenges of Wireless Sensor Network International Journal of Scientific Research in Computer Science, Engineering and Information Technology (c) 2018 IJSRCSEIT | Volume 3 | Issue 1 | ISSN : 2456- 3307 pp:746

[10] Madhava Rao K"Diverse Types Of Network Attacks And The Describing Security Mechanisms" International Journal of Engineering Science Invention (IJESI), vol. 07, no. 03, 2018, pp42-49

[11] Hyun Joo Park, Seong Cheol Kim, Hye Yun Kim.Delay Aware Data Gathering Mechanism in Wireless Sensor Networks.International Journal of Security Technology for Smart Device. Vol. 5. No. 2. Oct. 2018.GVPress. pp:15-20.http://dx.doi.org/10.21742/IJSTSD.2018.5.2.03

[12] Vempati Sudheshna.Adhoc Network Infrastructure Security.International Journal of Wireless and Mobile Communication for Industrial Systems. Vol. 3. No. 1. Apr. 2016.GVPress. pp:21-34. http://dx.doi.org/10.21742/IJWMCIS.2016.3.1.03

[13] Ashish Bagwari, Jyotshana Kanti and Geetam Singh Tomar.New Cooperative Spectrum Detection Technique in Cognitive Radio Networks.International Journal of Wireless and Mobile Communication For Industrial Systems. Vol. 3. No. 1. Apr. 2016.GVPress. pp:43 58.http://dx.doi.org/10.21742/IJWMCIS.2016.3.1.05

[14] SystemRekha Purohit and Prabhat Mathur.Role of Wireless Sensor Networks in Communication with Artificial Intelligence.International Journal of Wireless and Mobile Communication for Industrial Systems. Vol. 3. No. 2. Oct. 2016.GVPress. pp:33-38.http://dx.doi.org/10.21742/IJWMCIS.2016.3.2.05

[15] Shivangi Mishra, Priyanka Shrivastava and Priyanka Dhurvey.Change Detection Techniques in Remote Sensing: A Review.International Journal of Wireless and Mobile Communication for Industrial Systems. Vol. 4. No. 1. Apr. 2017.GVPress. pp:1-8.http://dx.doi.org/10.21742/IJWMCIS.2017.4.1.01 
International Journal of Security and Its Applications Vol. 13, No. 3 (2019) 